\section{New Frontiers in Ultrasonography of the Mediastinum: Pediatric EBUS-TBNA}

\section{To the Editor:}

I read with keen interest the review article by Fuso et $\mathrm{al}^{1}$ in which the authors performed a review on the endo-ultrasonographic modalities for evaluation of mediastinum. Although comprehensive, there are certain other areas where the use of endobronchial ultrasound-guided transbronchial needle aspiration (EBUS-TBNA) has revolutionized practice, and one such important indication is the utilization of EBUS-TBNA in children for the evaluation of undiagnosed mediastinal lymphadenopathy and masses. ${ }^{2,3}$

Prior to the pediatric application of EBUS-TBNA, evaluation of undiagnosed mediastinal lymphadenopathy in children required invasive surgical procedures like mediastinoscopy, thoracotomy, and thoracoscopic surgery. Due to the risk of morbidity with these invasive techniques, treatment was often initiated empirically on the basis of clinical radiological features, which has understandable limitations. Conventional blind TBNA in children has been rarely described and can be risky in the absence of real-time guidance during tracheobronchial needle puncture. ${ }^{4}$ EBUS-TBNA allows realtime visualization of the mediastinal lymph nodes during bronchoscopic needle aspiration and has evolved into a standard-of-care modality for the evaluation of mediastinal lymphadenopathy.

In the largest published series on pediatric EBUS-TBNA ( $N=67$ children), the sensitivity of the procedure in undiagnosed mediastinal lymphadenopathy was $79.1 \%$ and led to a meaningful change in diagnosis in $41.8 \%$ subjects. ${ }^{2}$ In addition, transesophageal introduction of the EBUS scope to perform needle aspiration (EUS-B-FNA) has advanced the frontiers of EBUS-TBNA even further. The first report on pediatric EUS-B-FNA described the use of this ap-

Correspondence: Karan Madan MD DM, Department of Pulmonary Medicine and Sleep Disorders, All India Institute of Medical Sciences, Ansari Nagar, New Delhi 110029, India. E-mail: drkaranmadan@gmail.com.

Dr Madan has disclosed no conflicts of interest.

DOI: $10.4187 /$ respcare.06739 proach to sample a subcarinal lymph node in a 3-year-old child in whom endotracheal introduction of the EBUS-TBNA scope was not feasible due to the smaller size of the pediatric trachea. ${ }^{5}$ Subsequently, this modality has been used safely and efficaciously to perform mediastinal lymph node sampling in children as young as 20 months. ${ }^{6}$ The transesophageal approach (EUS-BFNA) is particularly attractive as it obviates the need for tracheal entry and provides excellent access to the subcarinal (station 7) and left-sided (station 4L) mediastinal lymph nodes. Most of the published literature on pediatric EBUS has reported the use of either a $21 \mathrm{G}$ or a $22 \mathrm{G}$ needle for diagnosis. However, with the development of thinner needles (eg, 25G), the risk of anticipated complications is likely to be reduced even further, thus broadening the application of this procedure. This is important in light of the rapid developments in the field of cytodiagnostics.

The use of EBUS-TBNA in children has been found to be safe and can be performed under moderate sedation, and no major complications have been reported. EBUS-TBNA has a learning curve, and pediatric EBUSTBNA/EUS-B-FNA should be performed initially by experienced operators who are familiar with this procedure in adults whenever this modality is introduced in a new facility.

Karan Madan

Department of Pulmonary Medicine and Sleep Disorders

All India Institute of Medical Sciences Ansari Nagar, New Delhi, India

\section{REFERENCES}

1. Fuso L, Varone F, Magnini D, Calvello M, Greco EL, Richeldi L. Ultrasonography of the mediastinum: techniques, current practice, and future directions. Respir Care 2018; 63(11):1421-1438.

2. Dhooria S, Madan K, Pattabhiraman V, et al. A multicenter study on the utility and safety of EBUS-TBNA and EUS-B-FNA in children. Pediatr Pulmonol 2016;51(10): 1031-1039.

3. Madan K, Ayub II, Mohan A, Jain D, Guleria R, Kabra SK. Endobronchial ultrasoundguided transbronchial needle aspiration (EBUS-TBNA) in mediastinal lymphadenopathy. Indian J Pediatr 2015;82(4):378380.

4. Goussard P, Gie RP, Kling S, et al. The diagnostic value and safety of transbronchial needle aspiration biopsy in children with mediastinal lymphadenopathy. Pediatr Pulmonol 2010;45(12):1173-1179.

5. Madan K, Garg P, Kabra SK, Mohan A, Guleria R. Transesophageal bronchoscopic ultrasound-guided fine-needle aspiration (EUS-B-FNA) in a 3-year-old child. J Bronchology Interv Pulmonol 2015;22(4):347350 .

6. Mehta R, Biraris P, Shivakumar S, Misra S, Anoop P. Transesophageal bronchoscopic ultrasound-guided fine-needle aspiration (EUS-B-FNA): pushing the boundaries in the diagnosis. Pediatr Pulmonol 2017;52(11): E91-E93.

\section{New Frontiers in Ultrasonography of the Mediastinum: Pediatric EBUS-TBNA}

In Reply:

We read with great interest the letter by Dr Karan Madan on the application of endobronchial ultrasound-guided transbronchial needle aspiration (EBUS-TBNA) for the evaluation of undiagnosed mediastinal lymphadenopathy and masses in children. This letter is a useful complement to our review on the current practice and future perspectives of endoscopic ultrasound techniques in the evaluation of the mediastinum. ${ }^{1}$ Our review focused on the role of endo-ultrasonography in adults with both malignant and benign mediastinal diseases. Dr Madan has properly extended the topic to the pediatric population. In the letter, $\mathrm{Dr}$ Madan showed that the sensitivity of EBUSTBNA in children is almost as high as that obtained in adults. Moreover, the use of EBUS-TBNA in children has been found to be safe, with no major complications reported. Finally, the transesophageal approach to perform fine-needle aspiration (EUS-B-FNA) has further advanced the frontiers of EBUS-TBNA in pediatric population as well. We thank Dr Madan for giving us the opportunity to further emphasize the importance of endo-ultrasonographic techniques as first-choice tools for the diagnosis of mediastinal diseases both in adults and in children.

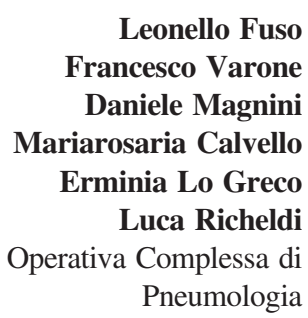




\section{LETTERS}

Università Cattolica del Sacro Cuore Fondazione Policlinico A. Gemelli,

IRCSS

Rome, Italy

\section{REFERENCE}

1. Fuso L, Varone F, Magnini D, Calvello M, Greco EL, Richeldi L. Ultrasonography of the mediastinum: techniques, current practice, and future directions. Respir Care 2018; 63(11):1421-1438.

The authors have disclosed no conflicts of interest.

DOI: $10.4187 /$ respcare.06739

\section{Music, Dance, and Harmonicas for People With COPD}

We read with great interest the paper by Hart et al, ${ }^{1}$ recently published in RESPIRATORY CARE, regarding the impact of playing the harmonica on outcomes for people with COPD. As part of the FRESH AIR project, ${ }^{2}$ we have been developing and delivering pulmonary rehabilitation programs in the Kyrgyz Republic, Uganda, Vietnam, and Greece. Our recent work in the Kyrgyz Republic highlighted the potential for integrating music and dance into pulmonary rehabilitation. We did not specifically explore the potential use of traditional wind instruments, but the findings of Hart et $\mathrm{al}^{1}$ highlight this as an area to explore.

In June 2018, we conducted stakeholder interviews in hospitals and rural clinics throughout the Kyrgyz Republic, during which we explored individual and site-specific views physical activity in chronic lung disease, and the potential use of music and dance to improve engagement in pulmonary rehabilitation. We conducted semistructured interviews and group discussions involving 42 subjects and 35 staff at 6 of the current trial sites of program delivery.

Because singing, music, and dance are major components of Kyrgyz culture, we explored perceptions on the potential use of music and dance as adjuncts to formal pulmonary rehabilitation (Fig. 1.). Responses from both subjects and staff at all sites were overwhelmingly positive, with comments such as "When we sing and dance, we live"; "When I dance, I stop thinking about my disease, and all the things that are wrong with me"; and "[Listening to] music takes me to another world.'

There was a clear and ubiquitous view that the addition of music, singing, and dancing would be feasible and welcome as adjuncts to the formal program as an engaging way to exercise, which might enhance participation, improve disease management, and boost mood and self-confidence. Few practical barriers to adding music and dance were mentioned. Some subjects also suggested that music could be played during the conventional exercise program, and at some sites this was already happening. On 4 occasions, discussions of singing, music, and dance resulted in group singing or dance sessions.

In summary, our findings highlight an eagerness to integrate music and dance into formal pulmonary rehabilitation programs in contexts outside the United Kingdom, such as the Kyrgyz Republic. The article by Hart et $\mathrm{al}^{1}$ further raises the potential of exploring the use of traditional instruments, which could provide further individualization and cultural adaptations of approaches to improve respiratory health.

Keir EJ Philip

Imperial College London

National Heart and Lung Institute London, United Kingdom

Azamat Akylbekov Bermet Stambaeva

Talant Sooronbaev

National Centre of Cardiology and Internal Medicine

Pulmonology Department Bishkek, Kyrgyz Republic

Rupert Jones

University of Plymouth

Faculty of Medicine and Dentistry Plymouth, United Kingdom

The authors have disclosed no conflicts of interest.

DOI: $10.4187 /$ respcare.06739

\section{REFERENCES}

1. Hart MK, Stewardson E, Jamil A, Tecson K, Millard M. Harmonica playing improves outcomes in patients with COPD. Respir Care 2018;63(Suppl 10):3024192.

2. International Primary Care Respiratory Group. FRESH AIR: An overview. Available at: https://www.theipcrg.org/display/DoResearch/ FRESH+AIR\%3A+An+overview. Accessed October. 16, 2018.

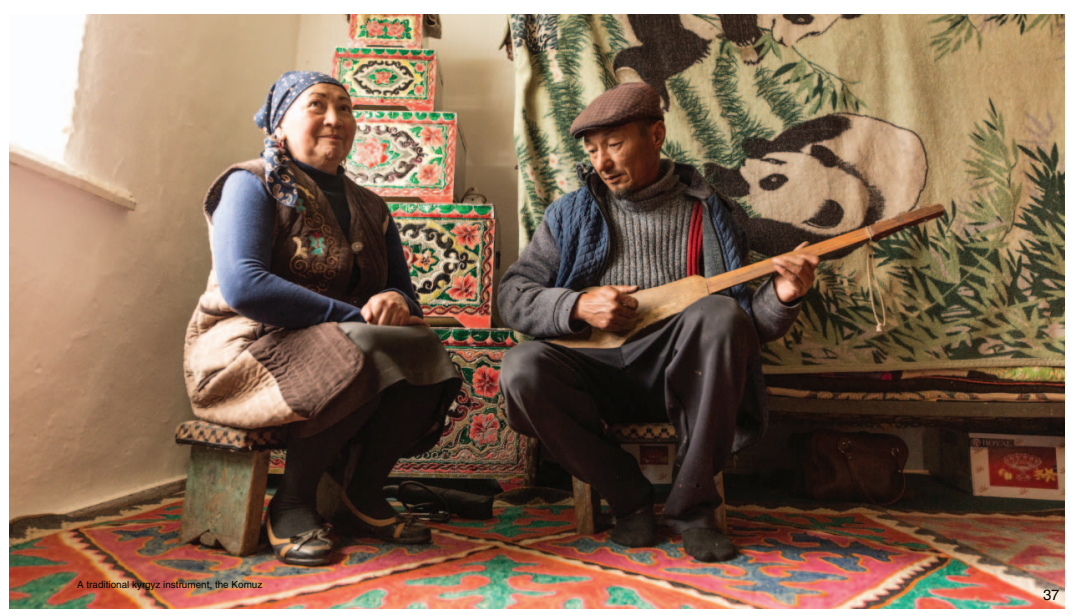

Fig. 1. A traditional Kyrgyz instrument, the komuz. Courtesy of Carey Marks. 